\title{
Haze Formation Limits the Primary Productivity of Marine Anaerobic Ecosystem during the Archean
}

\author{
YASUTO WATANABE ${ }^{1}$, EIICHI TAJIKA ${ }^{1}$, KAZUMI \\ OZAKI $^{2}$ AND PENG K. HONG ${ }^{3}$ \\ ${ }^{1}$ The University of Tokyo \\ ${ }^{2}$ Toho University \\ ${ }^{3}$ Chiba Institute of Technology \\ Presenting Author: yasuto@eps.s.u-tokyo.ac.jp
}

The marine primary production in the early Earth before the advent of oxygenic photosynthesis is suggested to have been limited by the supply of electron donors [1]. When the supply of electron donors is large enough to produce sufficient methane fluxes to the atmosphere, hydrocarbon haze is formed in the upper atmosphere. Because haze particles effectively absorb the UV-flux [2], it should affect the biological activity in the ocean via affecting the supply of electron donors produced in the atmosphere $\left(\mathrm{H}_{2}\right.$ and $\left.\mathrm{CO}\right)$. However, the relationship between the biological activity and the formation of haze has not been studied. Here, we employed a coupled model of one-dimensional photochemical model "Atmos" [3] and a marine microbial ecosystem model to estimate the impact of the formation of a haze layer to the marine anaerobic microbial ecosystem.

We found that the primary productivity of anaerobic biosphere decreases when the haze layer is formed with a large external input of the electron donor. This is because the production rate of the electron donor in the atmosphere is decreased because of the UV-shielding and the removal of haze particles. The productivity of anaerobic ecosystem is suppressed even with larger external input of electron donors because the haze layer thickens and the production rate of electron donors in the atmosphere decreases to adjust the increased external input. As a result, the maximum productivity of anaerobic microbial ecosystem is achieved at the clear-sky condition at the boundary of haze formation. This maximum level is around $1.5 \mathrm{GtC} / \mathrm{yr}$, irrespective of the atmospheric $\mathrm{CO}_{2}$ levels and of the combinations of external input of electron donors (volcanic $\mathrm{H}_{2}$ outgassing flux and ferrous iron upwelling flux). Thus, haze formation provides a concrete upper limit in the primary productivity of marine anaerobic microbial ecosystem during the Archean. Our findings also constrain the possibility of a hazy atmosphere during the Archean.

[1] Kharecha et al., 2005, Geobiology, 3(2): 53-76.

[2] Wolf and Toon, 2010, Science 328 (5983): 1266-68.

[3] Arney et al. 2016, Astrobiology 16 (11): 873-99. 